\title{
Quality of some hybrid seeds of rice and control of seed borne fungi in Bangladesh
}

\author{
M. A. Akter and I. Hossain* \\ Department of Plant Pathology, Bangladesh Agricultural University, Mymensingh-2202, Bangladesh \\ *E-mail: dhossain69 @gmail.com
}

\begin{abstract}
A study was conducted for assessing quality of 15 hybrid rice seed samples and management of seed borne fungal pathogens by three selected chemicals namely Bavistin (0.3\%), Thiovit (0.3\%) and Provax (0.3\%) along with two botanicals such as: Garlic extract (Allium sativum) (1:1) and Allamanda leaf extract (Allamanda cathartica) (1:1) and a bio-control agent BAU-Biofungicide @ 3\% (Trichoderma based preparation) at Seed Pathology Centre, MS Laboratory and Eco-friendly Plant Disease Management Laboratory of Department of Plant Pathology, Bangladesh Agricultural University, Mymensingh during 2013-14. Collected seed samples were categorized by dry inspection as apparently healthy seed (35.7 to $85.7 \%)$, discolored seed $(0.5$ to $22.2 \%)$, spotted seed (0.7 to $8.1 \%)$, broken seed (0.4 to $44.3 \%)$, deformed seed (0 to $1.4 \%$ ), insect damaged seed (0 to $4.9 \%$ ), chaffy seed (0 to $1.1 \%)$, varietal mixture (0 to $7.2 \%$ ) and inert matter (0 to 2.6\%). In case of purity analysis, pure seed ranged from 92.86 to $100.00 \%$, other seeds 0 to $7.14 \%$ and inert matter 0 to $2.59 \% .100 \%$ pure seed was recorded in Agomoni, Jagoron, Gold, Suborno, Hira-1, Hira-2, Hira-4 and Hira-6 collected from Ispahani Agro. Ltd., BRAC, Lal Teer seed Co. Ltd. and Supreme seed Co. Ltd. Seed treatment with Allamanda leaf extract, Provax and BAU-Biofungicide increased germination, Vigor index and decreased prevalence of seed borne fungi. Among the treatments, biofungicide increased germination $54.39 \%$ in blotter method and $89.58 \%$ in tray method over control. In addition, BAUBiofungicide increased up to $713.78 \%$ vigor index over untreated control. BAU-Biofungicide may be recommended for controlling seed borne fungal pathogens of hybrid rice in Bangladesh.
\end{abstract}

Keywords: Hybrid Rice, Seed purity, Seed borne pathogens, Botanical extract, Biological control agent

\section{Introduction}

Rice (Oryza sativa L.), belongs to the family Graminae, is one of the most important cereals in the world. It is the most important food for over two billion peoples in Asia and for hundreds of millions in Africa and Latin America. To feed the ever increasing population of these regions the world's annual rice production must be increased from the present 560 to 750 million tons by 2020 (Saranraj et al., 2013). Rice is the staple food of Bangladeshi people and it constituted about $90 \%$ of the total food grain production (Ahmed et al. 2013). The average production per hectare of rice in Bangladesh is low as compared to other rice growing countries of the world. Seed is one of the most important technologies for crop production. For successful crop production there is no other alternative but to use good seed. It has been shown experimentally that only by using good quality healthy seed, rice yield could be increased by $10-15 \%$ (Akter et al., 2015). The yield of conventional rice varieties is comparatively low and it seems impossible to change this yield with reachable resources under the prevailing situation. At this situation hybrid rice varieties may be a break through which could help to achieve the goal of self-sufficiency of food. In Bangladesh, more than 78 hybrid rice varieties are grown in the field and about $75 \%$ of seed demand is met from import mostly from China (Ora et al. 2011). In Bangladesh, approximately 2.5 million tons of rice worth more than Tk. 12000 Millions lost annually due to diseases caused by seed borne pathogens (Fakir et al. 2003). Rice suffers from more than 60 different diseases. In Bangladesh, 43 diseases are known to occur on the rice crop. Among these diseases, 27 are seed borne of which 14 are of major importance. Fungi are the principal organisms associated with seed in storage. Of all the seed borne diseases of rice, 22 are caused by fungi (Fakir, 2000). High quality seed means the seed must be genetically pure with high germination capacity and high yield potential. Although the seed system in Bangladesh is at a very rudimentary stage, a total of 5 lac tons of seeds including the seeds of cereals and other crops per year is required, out of which only $18 \%$ seeds are produced by different seed organizations with care but almost regardless of the health status (Hossain and Dey, 2011). It is incontestable that proper seed treatment measures can substantially improve the quality of seed and significantly increase the yield. On the other 
hand, to ensure eco-friendly disease management, Biological and botanicals are using instead of hazardous chemicals. BAU-Biofungicide, a biological control agent in Bangladesh resulted significant higher germination and plant stand, less disease incidence and higher yield of different crops (Hossain, 2011), Chowdhury et al. (2013), Hossain and Hossain (2012). Until now considerable work has been done on seed quality of rice by different researchers in the different regions of Bangladesh. However, information about the health and quality of hybrid rice seed of Bangladesh is rare. Therefore, the present study was undertaken to investigate the health and quality of hybrid seeds of rice and to control the seed borne pathogens of hybrid rice seeds by chemical, botanical and biological means.

\section{Materials and Methods}

The experiment pertaining to the present investigation was carried out in the Seed Pathology Centre (SPC), Bangladesh Agricultural University (BAU), Mymensingh; Eco-friendly Plant Disease Management Laboratory and MS Laboratory of the Department of Plant Pathology, BAU, Mymensingh for studying health and quality of seed samples of 15 hybrid rice varieties. In addition control of seed borne fungal pathogens was done in this study. Seed samples were dry inspected according to ISTA Rules (1996) and graded into nine categories viz i) apparently healthy seed, ii) broken seeds, iii) spotted seeds, iv) discolored seeds, v) deformed seeds, vi) insect damaged seeds, vii) chaffy seeds, viii) varietal mixture and ix) inert matter. Purity analysis of each sample was done following standard procedure for purity test (ISTA, 1996). Three different types of chemical fungicides viz. Bavistin (0.3\%), Thiovit (0.3\%) and Provax (0.3\%), two botanicals viz. Garlic extract (Allium sativum) (1:1) and Allamanda leaf extract (Allamanda cathartica) (1:1) and a biological seed treating agent viz. BAU-Biofungicide (3\%) were used for controlling seed borne fungal pathogens. BAU-Biofungicide, a Trichoderma based preparation (Hossain, 2011). The seeds were treated by soaking in solutions for $1 \mathrm{hr}$ of each treatment separately, except in control. In case of control, seeds were soaked in normal water.After 1 hour the fungicidal solutions were drained out and the moistened seeds were kept in the blotter paper to remove excess moisture from the seed surface. Then the seeds were ready for placing on blotter paper (blotter method) and tray experiment. Seeds were set for each treatment according to ISTA rules for testing germination of seeds (ISTA, 2001). Germination was recorded twice at 7 and 14 days after sowing. Total germinated seeds were counted and expressed in percentage. Vigor test was also done according to ISTA (2001). Shoot and root length were measured by using measuring scale after 14 days of placing. Fifteen seedlings (30 seedlings of each treatment) were randomly selected for the measurement of shoot and root length. The seedling vigor was determined following the formula of Baki and Anderson (1972):

Vigor index $=$ (mean of root length + mean of shoot length $) \times \%$ of seed germination .

The data collected from the experiments were analyzed for test of significance and compared the treatment means by using Duncan's Multiple Range Test (DMRT) at $5 \%$ level of probability following the MSTAT-C program.

\section{Results and Discussion}

The results of dry inspection of 15 cultivars of the collected hybrid rice seeds of different seed companies are presented in Table 1 . Collected seed samples were categorized by dry inspection as apparently healthy seed (35.7 to $85.7 \%)$, discolored seed (0.5 to $22.2 \%)$, spotted seed (0.7 to $8.1 \%)$, broken seed (0.4 to $44.3 \%$ ), deformed seed (0 to $1.4 \%$ ), insect damaged seed (0 to $4.9 \%$ ), chaffy seed (0 to $1.1 \%$ ), varietal mixture (0 to $7.2 \%$ ) and inert matter (0 to 2.6\%). The highest number of apparently healthy seed (85.7\%) was recorded from variety Tia of Lal Teer Seed Co. Ltd. and lowest (35.7\%) from Safollo collected from Supreme Seed Co. Ltd. Fakir et al. (2002) studied rice seed sample in Bangladesh and recorded 91.20 to $98.89 \%$ pure seed, 3.72 to $37.71 \%$ spotted seed, $8.46-15.50 \%$ deformed seed collected from Rajshahi, Rangpur and Bogra of Bangladesh. Fakir et al. (2003) also recorded wide variation of components among the collected seed samples of rice from different regions of Bangladesh. Nahar et al. (2004) studied with Farmers stored rice seeds of Bogra district and found apparently healthy seed, spotted seeds, deformed seeds, discolored seeds, chaffy grains and insect damaged seeds by $77.84 \%, 16.32 \%, 3.22 \%, 1.03 \%$ and $0.56 \%$, respectively. Uddin (2005) studied the Farmer seeds of 
Begumganj Upazilla in Noakhali, and recorded apparently healthy seed (44.33 to $59.42 \%$ ), spotted seed (27.84 to $44.77 \%)$, discolored seed (3.93 to $8.94 \%)$, partly filled seed $(0.43$ to $2.35 \%)$, deformed seed (0.91 to $3.98 \%)$, unfilled seed $(0.001$ to $0.68 \%)$, varietal mixture $(0.26$ to $2.22 \%)$, other plant parts $(0.001$ to $0.36 \%)$, inert matter (0.50 to $0.34 \%)$ and insect damaged seed (0.05 to $0.75 \%)$. From the above findings it is clear that seeds of variety Tia of Lal Teer Seed Co. Ltd. was found superior over all other tested hybrid seeds.

Table 1. Dry inspection of hybrid rice seed samples collected from different seed companies of Bangladesh

\begin{tabular}{cll|l|c|c|c|c|c|c|c|c|c}
\hline Sl. N. & Variety & Seed source & \multicolumn{9}{|c|}{ Different grades of seeds (\%) } & \\
\cline { 4 - 10 } & & & & $\begin{array}{c}\text { Apparently } \\
\text { healthy } \\
\text { seed }\end{array}$ & $\begin{array}{c}\text { Discolored } \\
\text { Seed }\end{array}$ & $\begin{array}{c}\text { Spotted } \\
\text { seed }\end{array}$ & $\begin{array}{c}\text { Broken } \\
\text { seed }\end{array}$ & $\begin{array}{c}\text { Insect } \\
\text { damaged } \\
\text { seed }\end{array}$ & $\begin{array}{c}\text { Chaffy } \\
\text { seed }\end{array}$ & $\begin{array}{c}\text { Deformed } \\
\text { seed }\end{array}$ & $\begin{array}{c}\text { Varietal } \\
\text { Mixture }\end{array}$ & $\begin{array}{c}\text { Inert } \\
\text { matter }\end{array}$ \\
\hline 1 & Durber & Ispahani Agro. Ltd. & 57.3 & 14.8 & 2.2 & 24.3 & 0.0 & 0.4 & 0.5 & 0.5 & 0.0 \\
2 & Agomoni & Ispahani Agro. Ltd. & 56.3 & 9.3 & 3.8 & 27.3 & 2.9 & 0.1 & 0.26 & 0.0 & 0.0 \\
3 & Meghna & Ispahani Agro. Ltd. & 41.2 & 13.4 & 3.6 & 41.4 & 0.3 & 0.0 & 0.0 & 0.2 & 0.0 \\
4 & Hybrid super & BADC & 85.4 & 11.6 & 2.2 & 0.4 & 0.2 & 0.0 & 0.0 & 0.0 & 0.2 \\
5 & Aloron & BRAC & 55.1 & 17.6 & 1.8 & 16.3 & 4.9 & 0.4 & 0.6 & 0.4 & 2.6 \\
6 & Jagoron & BRAC & 49.7 & 22.2 & 3.2 & 23.7 & 0.4 & 0.6 & 0.2 & 0.0 & 0.0 \\
7 & Moyna & LalTeer seed co. Ltd. & 61.4 & 5.7 & 1.5 & 28.6 & 0.7 & 0.0 & 0.0 & 2.2 & 0.0 \\
8 & Tia & LalTeer seed co. Ltd. & 85.7 & 4.3 & 0.7 & 8.6 & 0.0 & 0.0 & 0.0 & 0.7 & 0.0 \\
9 & Gold & LalTeer seed co. Ltd. & 68.5 & 3.6 & 7.2 & 27.2 & 0.0 & 0.0 & 0.0 & 0.0 & 0.0 \\
10 & Safollo & Supreme seed co. Ltd. & 35.7 & 8.6 & 4.3 & 44.3 & 0.0 & 0.0 & 0.0 & 7.2 & 0.0 \\
11 & Suborno & Supreme seed co. Ltd. & 64.3 & 15.7 & 2.9 & 15.7 & 0.0 & 0.0 & 1.4 & 0.0 & 0.0 \\
12 & Hira-1 & Supreme seed co. Ltd. & 74.3 & 10 & 4.3 & 11.5 & 0.0 & 0.0 & 0.0 & 0.0 & 0.0 \\
13 & Hira-2 & Supreme seed co. Ltd. & 74.3 & 11.4 & 2.9 & 11.5 & 0.0 & 0.0 & 0.0 & 0.0 & 0.0 \\
14 & Hira-4 & Supreme seed co. Ltd. & 83.8 & 0.5 & 8.1 & 5.5 & 0.2 & 1.1 & 0.9 & 0.0 & 0.0 \\
15 & Hira-6 & Supreme seed co. Ltd. & 64.3 & 15.7 & 2.9 & 15.7 & 0.0 & 0.0 & 1.4 & 0.0 \\
\hline
\end{tabular}

Apparently healthy seed $=$ Good looking seed free from spots and abnormalities.

In case of purity analysis, pure seed of hybrid seed samples ranged from 92.86 to $100.00 \%$, other seeds 0 to $7.14 \%$ and inert matter 0 to $2.59 \%$ (Table 2). 100\% pure seed was recorded in 8 hybrid rice varieties including 4 seed companies. The lowest pure seed (92.86\%) was recorded in variety Safollo collected from Supreme Seed Co. Ltd (Table 2). Chowdhury (2012) studied seed quality status of HYV rice seed in Bangladesh. Haque et al. (2007) found $99.01 \%$ pure seed from the farmers produced rice seeds in seed samples of trained Farmers and minimum (96.19\%) in untrained Farmers. Uddin (2005) determined the percentage of pure seed of Begumgonj Upazilla under Noakhali district that ranged from 95.59 to $99.39 \%$. Fakir et al. (2002) reported the percentage of pure seeds ranging from 91.20 to $98.89 \%$ collected from farmers stored rice seed. It is clear that the purity of company hybrid seeds is comparatively higher than farmers stored rice seeds. Out of the seed sample tested, seeds of eight hybrid seeds (Agomoni, Jagoron, Gold, Suborno, Hira-1, Hira-2, Hira-4 and Hira-6) were found 100\% pure.

Means of \% germination of different hybrid seeds ranged from $53.0 \%$ to $97.5 \%$. The highest germination (97.5\%) was recorded in variety Agomoni treated with BAU-Biofungicide. The lowest germination (53.0\%) was found in Control of variety Tia. The highest increase of germination (54.39\%) over control was recorded when seeds treated with BAU-Biofungicide which was followed by Allamanda leaf extract (Table 3). The lowest increase of germination (13.89\%) over control was recorded in case of seeds treated with Garlic extract followed by Bavistin. Sultana et al. (2009) and Hossain (2011) reported that BAU Bio-fungicide increased germination and seedling vigor of some vegetables seeds. Howlader (2003) reported that seed treatment with Allamanda leaf extract (1:1) effectively increased germination of egg plant seeds and decreased nursery diseases. The present study clearly showed that BAU-Biofungicide resulted superior effect in increasing germination of seeds. 
Table 2. Purity analysis of hybrid rice seeds collected from different seed companies of Bangladesh

\begin{tabular}{|c|c|c|c|c|c|}
\hline \multirow[b]{2}{*}{ SI. No. } & \multirow[b]{2}{*}{ Variety } & \multirow[b]{2}{*}{ Seed source } & \multicolumn{3}{|c|}{ Different components under purity analysis (\%) } \\
\hline & & & Pure Seed & Other seed & Inert matteI \\
\hline 1 & Durber & Ispahani Agro. Ltd. & 99.52 & 0.48 & 0.00 \\
\hline 2 & Agomoni & Ispahani Agro. Ltd. & 100.00 & 0.00 & 0.00 \\
\hline 3 & Meghna & Ispahani Agro. Ltd. & 99.80 & 0.20 & 0.00 \\
\hline 4 & Hybrid super & BADC & 99.82 & 0.00 & 0.18 \\
\hline 5 & Aloron & BRAC & 97.04 & 0.37 & 2.59 \\
\hline 6 & Jagoron & BRAC & 100.00 & 0.00 & 0.00 \\
\hline 7 & Moyna & LalTeer Seed Co. Ltd. & 97.85 & 2.14 & 0.00 \\
\hline 8 & Tia & LalTeer Seed Co. Ltd. & 99.29 & 0.71 & 0.00 \\
\hline 9 & Gold & LalTeer Seed Co. Ltd. & 100.00 & 0.00 & 0.00 \\
\hline 10 & Safollo & Supreme Seed Co. Ltd. & 92.86 & 7.14 & 0.00 \\
\hline 11 & Suborno & Supreme Seed Co. Ltd. & 100.00 & 0.00 & 0.00 \\
\hline 12 & Hira-1 & Supreme Seed Co. Ltd. & 100.00 & 0.00 & 0.00 \\
\hline 13 & Hira-2 & Supreme Seed Co. Ltd. & 100.00 & 0.00 & 0.00 \\
\hline 14 & Hira-4 & Supreme Seed Co. Ltd. & 100.00 & 0.00 & 0.00 \\
\hline 15 & Hira-6 & Supreme Seed Co. Ltd. & 100.00 & 0.00 & 0.00 \\
\hline
\end{tabular}

Total seed counted $=70 \mathrm{~g}$ as per rules of ISTA (1996)

Table 3. Effect of seed treatment on germination of different varieties of hybrid rice by Blotter method

\begin{tabular}{|c|c|c|c|c|c|c|c|c|}
\hline \multirow[t]{3}{*}{ SI No. } & \multirow[t]{3}{*}{ Variety } & \multicolumn{7}{|c|}{ \% germination } \\
\hline & & \multicolumn{7}{|c|}{ Treatment } \\
\hline & & $T_{1}$ & $T_{2}$ & $T_{3}$ & $\mathrm{~T}_{4}$ & $T_{5}$ & $T_{6}$ & $T_{7}$ \\
\hline 1. & Durber & $\begin{array}{c}93.0 \\
(30.99)\end{array}$ & $\begin{array}{c}90.0 \\
(26.77)\end{array}$ & $91.0(28.17)$ & $91.0(28.17)$ & $\begin{array}{c}87.0 \\
(22.54)\end{array}$ & $\begin{array}{c}93.0 \\
(30.99)\end{array}$ & 71.0 \\
\hline 2. & Agomoni & $\begin{array}{c}97.5 \\
(40.29)\end{array}$ & $\begin{array}{c}91.0 \\
(30.94)\end{array}$ & $\begin{array}{c}92.0 \\
(32.37)\end{array}$ & $90.0(29.50)$ & $\begin{array}{c}81.0 \\
(16.55)\end{array}$ & $\begin{array}{c}95.0 \\
(36.70)\end{array}$ & 69.5 \\
\hline 3. & Meghna & $\begin{array}{c}90.0 \\
(48.77)\end{array}$ & $\begin{array}{c}90.0 \\
(48.77)\end{array}$ & $90.0(48.77)$ & $90.0(48.77)$ & $\begin{array}{c}80.0 \\
(32.23)\end{array}$ & $\begin{array}{c}90.0 \\
(48.77)\end{array}$ & 60.5 \\
\hline 4. & Hybrid super & $\begin{array}{c}93.5 \\
(52.03)\end{array}$ & $\begin{array}{c}89.0 \\
(44.72)\end{array}$ & $90.0(46.34)$ & $90.0(46.34)$ & $\begin{array}{c}82.0 \\
(33.33)\end{array}$ & $\begin{array}{c}91.5 \\
(48.79)\end{array}$ & 61.5 \\
\hline 5. & Aloron & $\begin{array}{c}83.0 \\
(50.91)\end{array}$ & $\begin{array}{c}84.0 \\
(52.73)\end{array}$ & $82.0(49.10)$ & $82.0(49.10)$ & $\begin{array}{c}81.0 \\
(47.27)\end{array}$ & $\begin{array}{c}\text { 83.(50.91 } \\
\text { 0 }\end{array}$ & 55.0 \\
\hline 6. & Jagoron & $\begin{array}{c}96.5 \\
(46.21)\end{array}$ & $\begin{array}{c}85.5 \\
(29.55)\end{array}$ & $90.0(36.36)$ & $90.0(36.36)$ & $\begin{array}{c}80.0 \\
(21.21)\end{array}$ & $\begin{array}{c}95.0 \\
(43.94)\end{array}$ & 66.0 \\
\hline 7. & Moyna & $\begin{array}{c}80.0 \\
(42.86)\end{array}$ & $\begin{array}{c}83.0 \\
(48.21)\end{array}$ & 79.9 (42.68) & $80.0(42.86)$ & $\begin{array}{c}80.0 \\
(42.86)\end{array}$ & $\begin{array}{c}80.0 \\
(42.86)\end{array}$ & 56.0 \\
\hline 8. & Tia & $\begin{array}{c}80.0 \\
(50.94)\end{array}$ & $\begin{array}{c}79.5 \\
(50.00)\end{array}$ & $80.0(50.94)$ & $81.0(52.83)$ & $\begin{array}{c}75.0 \\
(41.51)\end{array}$ & $\begin{array}{c}80.0 \\
(50.94)\end{array}$ & 53.0 \\
\hline 9. & Gold & $\begin{array}{c}88.0 \\
(54.39)\end{array}$ & $\begin{array}{c}80.0 \\
(40.35)\end{array}$ & $81.0(42.11)$ & $82.0(43.86)$ & $\begin{array}{c}80.0 \\
(40.35)\end{array}$ & $\begin{array}{c}87.0 \\
(52.63)\end{array}$ & 57.0 \\
\hline 10. & Safollo & $\begin{array}{c}91.0 \\
(42.19)\end{array}$ & $\begin{array}{c}82.0 \\
(28.13)\end{array}$ & $84.0(31.25)$ & $87.0(35.94)$ & $\begin{array}{c}81.0 \\
(26.56)\end{array}$ & $\begin{array}{c}90.0 \\
(40.63)\end{array}$ & 64.0 \\
\hline 11. & Suborno & $\begin{array}{c}93.0 \\
(50.00)\end{array}$ & $\begin{array}{c}81.0 \\
(30.65)\end{array}$ & $83.0(33.87)$ & $85.5(37.90)$ & $\begin{array}{c}80.0 \\
(29.03)\end{array}$ & $\begin{array}{c}91.0 \\
(46.77)\end{array}$ & 62.0 \\
\hline 12. & Hira-1 & $\begin{array}{c}97.0 \\
(36.62)\end{array}$ & $\begin{array}{c}83.0 \\
(16.90)\end{array}$ & $90.0(26.76)$ & $90.0(26.76)$ & $\begin{array}{c}81.0 \\
(14.08)\end{array}$ & $\begin{array}{c}95.0 \\
(33.80)\end{array}$ & 71.0 \\
\hline 13. & Hira-2 & $\begin{array}{c}95.0 \\
(31.95)\end{array}$ & $\begin{array}{c}88.5 \\
(22.92)\end{array}$ & $90.0(25.00)$ & $91.0(26.39)$ & $\begin{array}{c}82.0 \\
(13.89)\end{array}$ & $\begin{array}{c}91.0 \\
(26.39)\end{array}$ & 72.0 \\
\hline 14. & Hira-4 & $\begin{array}{c}95.5 \\
(51.59)\end{array}$ & $\begin{array}{c}85.5 \\
(35.72)\end{array}$ & 89.5 (42.06) & $89.5(42.06)$ & $\begin{array}{c}80.0 \\
(26.98)\end{array}$ & $\begin{array}{c}93.5 \\
(48.41)\end{array}$ & 63.0 \\
\hline 15. & Hira-6 & $\begin{array}{c}93.0 \\
(36.76)\end{array}$ & $\begin{array}{c}86.0 \\
(26.47)\end{array}$ & $88.0(29.41)$ & $89.0(30.89)$ & $\begin{array}{c}82.0 \\
(20.59)\end{array}$ & $\begin{array}{c}90.0 \\
(32.35)\end{array}$ & 68.0 \\
\hline
\end{tabular}

Data in parenthesis indicate \% increase over control.

$\mathrm{T}_{1}=\mathrm{BAU}-$ Biofungicide (3\%), $\mathrm{T}_{2}=$ Bavistin (0.3\%), $\mathrm{T}_{3}=$ Thiovit $(0.3 \%), \mathrm{T}_{4}=$ Provax (0.3\%), $\mathrm{T}_{5}=$ Garlic extract $(1: 1), \mathrm{T}_{6}=$ Allamanda leaf extract (1:1) and $\mathrm{T}_{7}=$ Control (untreated) 
Means germination percentage for these hybrid rice samples ranged from $43.0 \%$ to $98.00 \%$ (Table 4). The highest (98.00\%) germination was recorded in case of seed treated with BAU Bio-fungicide of variety Meghna, Hybrid Super, Hira-1, Hira-2, Hira-4 and Hira-6 which was followed by Allamanda leaf extract. The lowest (43.0\%) germination was found in Control (untreated) of variety Aloron. Seed treatment with BAU Bio-fungicide, Bavistin, Thiovit, Provax, Garlic extract and Allamanda leaf extract showed significant effect on increasing germination percentage of the used hybrid rice samples. The highest \% increase of germination over control was recorded in case of seeds treated with BAU Bio-fungicide which was followed by Allamanda leaf extract (Table 4). Upto 89.58\% higher germination over control was achieved by treating seeds with BAU Bio-fungicide. Naznin and Hossain (2004) studied the effect of BAU Biofungicide on germination of some vegetables and showed that in sweet gourd, BAU-Biofungicide increased germination up to $5.22 \%$ in pot and $46.25 \%$ in field over the untreated control, while in cowpea, cucumber and okra the germination increased up to $46.245,7.92 \%$ and $50.80 \%$, respectively in pot experiment and $102.95,28.8 \%$ and $47.8 \%$ in field trial. Sultana et al. (2009) used BAU Bio-fungicide for seed treatment to control Bipolaris sorokiniana, leaf spot of wheat in cv. Kanchan. and observed (94\%) germination which $12.7 \%$ higher over control. They reported that Trichoderma based preparation increased germination of seeds.

Table 4. Effect of seed treatment on seed germination of different varieties of hybrid rice by Sand culture method

\begin{tabular}{|c|c|c|c|c|c|c|c|c|}
\hline \multirow[t]{3}{*}{ SI No. } & \multirow[t]{3}{*}{ Variety } & \multicolumn{7}{|c|}{$\%$ seed germination } \\
\hline & & \multicolumn{7}{|c|}{ Treatment } \\
\hline & & $T_{1}$ & $T_{2}$ & $T_{3}$ & $T_{4}$ & $T_{5}$ & $T_{6}$ & $T_{7}$ \\
\hline 1. & Durber & $\begin{array}{l}93.00 \mathrm{a} \\
(69.10)\end{array}$ & $\begin{array}{l}75.00 \mathrm{c} \\
(36.37)\end{array}$ & $\begin{array}{l}82.00 \mathrm{bc} \\
(49.10)\end{array}$ & $\begin{array}{l}86.00 \mathrm{ab} \\
(56.37)\end{array}$ & $\begin{array}{l}74.00 \mathrm{c} \\
(34.55)\end{array}$ & $\begin{array}{l}89.00 \mathrm{ab} \\
(61.82)\end{array}$ & $55.00 \mathrm{~d}$ \\
\hline 2. & Agomoni & $\begin{array}{l}82.00 \mathrm{a} \\
(60.78)\end{array}$ & $\begin{array}{l}70.00 \mathrm{ab} \\
(37.25)\end{array}$ & $\begin{array}{l}73.00 \mathrm{ab} \\
(43.14)\end{array}$ & $\begin{array}{l}76.00 \mathrm{ab} \\
(49.02)\end{array}$ & $\begin{array}{l}67.00 \mathrm{~b} \\
(31.37)\end{array}$ & $\begin{array}{l}82.00 \mathrm{a} \\
(60.78)\end{array}$ & $51.00 \mathrm{c}$ \\
\hline 3. & Meghna & $\begin{array}{l}98.00 \mathrm{a} \\
(28.95)\end{array}$ & $\begin{array}{l}91.00 \mathrm{a} \\
(19.74)\end{array}$ & $\begin{array}{l}95.00 \mathrm{a} \\
(25.00)\end{array}$ & $\begin{array}{l}92.00 \mathrm{a} \\
(21.05)\end{array}$ & $\begin{array}{l}91.00 \mathrm{a} \\
(19.74)\end{array}$ & $\begin{array}{l}96.00 \mathrm{a} \\
(26.32)\end{array}$ & $76.00 \mathrm{~b}$ \\
\hline 4. & Hybrid super & $\begin{array}{l}98.00 \mathrm{a} \\
(28.95)\end{array}$ & $\begin{array}{l}91.00 \mathrm{a} \\
(19.74)\end{array}$ & $\begin{array}{l}95.00 \mathrm{a} \\
(25.00)\end{array}$ & $\begin{array}{l}92.00 \mathrm{a} \\
(21.05)\end{array}$ & $\begin{array}{l}91.00 \mathrm{a} \\
(19.74)\end{array}$ & $\begin{array}{l}96.00 \mathrm{a} \\
(26.32)\end{array}$ & $76.00 \mathrm{~b}$ \\
\hline 5. & Aloron & $\begin{array}{l}75.00 \mathrm{a} \\
(74.42)\end{array}$ & $\begin{array}{l}56.00 \mathrm{c} \\
(30.23)\end{array}$ & $\begin{array}{l}59.00 \mathrm{bc} \\
(37.21)\end{array}$ & $\begin{array}{l}71.00 \mathrm{ab} \\
(65.12)\end{array}$ & $\begin{array}{l}56.00 \mathrm{c} \\
(30.23)\end{array}$ & $\begin{array}{l}62.00 a b c \\
(44.19)\end{array}$ & $43.00 \mathrm{~d}$ \\
\hline 6. & Jagoron & $\begin{array}{l}93.00 \mathrm{a} \\
(78.85)\end{array}$ & $\begin{array}{l}93.00 \mathrm{a} \\
(78.85)\end{array}$ & $\begin{array}{l}88.00 \mathrm{a} \\
(69.23)\end{array}$ & $\begin{array}{l}92.00 \mathrm{a} \\
(76.92)\end{array}$ & $\begin{array}{l}90.00 \mathrm{a} \\
(73.08)\end{array}$ & $\begin{array}{l}88.00 \mathrm{a} \\
(69.23)\end{array}$ & $52.00 \mathrm{~b}$ \\
\hline 7. & Moyna & $\begin{array}{l}90.00 \mathrm{a} \\
(83.67)\end{array}$ & $\begin{array}{l}82.00 \mathrm{~b} \\
(67.35)\end{array}$ & $\begin{array}{l}80.00 \mathrm{~b} \\
(63.27)\end{array}$ & $\begin{array}{l}82.00 \mathrm{~b} \\
(67.35)\end{array}$ & $\begin{array}{l}81.00 \mathrm{~b} \\
(65.31)\end{array}$ & $\begin{array}{l}91.00 \mathrm{a} \\
(85.71)\end{array}$ & $49.00 \mathrm{c}$ \\
\hline 8. & Tia & $\begin{array}{l}89.00 \mathrm{a} \\
(81.63)\end{array}$ & $\begin{array}{l}83.00 a b \\
(69.39)\end{array}$ & $\begin{array}{l}84.00 \mathrm{ab} \\
(71.42)\end{array}$ & $\begin{array}{l}85.00 \mathrm{a} \\
(73.47)\end{array}$ & $\begin{array}{l}77.00 \mathrm{~b} \\
(57.14)\end{array}$ & $\begin{array}{l}89.00 \mathrm{a} \\
(81.63)\end{array}$ & $49.00 \mathrm{c}$ \\
\hline 9. & Gold & $\begin{array}{l}91.00 \mathrm{a} \\
(89.58)\end{array}$ & $\begin{array}{l}83.00 \mathrm{bc} \\
(72.92)\end{array}$ & $\begin{array}{l}84.00 \mathrm{abc} \\
(75.00)\end{array}$ & $\begin{array}{l}85.00 \mathrm{abc} \\
(77.10)\end{array}$ & $\begin{array}{l}78.00 \mathrm{c} \\
(62.50)\end{array}$ & $\begin{array}{l}90.00 \mathrm{ab} \\
(87.50)\end{array}$ & $48.00 \mathrm{~d}$ \\
\hline 10. & Safollo & $\begin{array}{l}97.00 \mathrm{a} \\
(64.41)\end{array}$ & $\begin{array}{l}92.00 \mathrm{ab} \\
(55.93)\end{array}$ & $\begin{array}{l}91.00 \mathrm{ab} \\
(54.24)\end{array}$ & $\begin{array}{l}90.00 \mathrm{ab} \\
(52.54)\end{array}$ & $\begin{array}{l}84.00 \mathrm{~b} \\
(42.37)\end{array}$ & $\begin{array}{l}98.00 \mathrm{a} \\
(66.10)\end{array}$ & $59.00 \mathrm{c}$ \\
\hline 11. & Suborno & $\begin{array}{l}97.00 \mathrm{a} \\
(64.41)\end{array}$ & $\begin{array}{l}92.00 \mathrm{ab} \\
(55.93)\end{array}$ & $\begin{array}{l}91.00 \mathrm{ab} \\
(54.24)\end{array}$ & $\begin{array}{l}90.00 \mathrm{ab} \\
(52.54)\end{array}$ & $\begin{array}{l}84.00 \mathrm{~b} \\
(42.37)\end{array}$ & $\begin{array}{l}98.00 \mathrm{a} \\
(66.10)\end{array}$ & $59.00 \mathrm{c}$ \\
\hline 12. & Hira-1 & $\begin{array}{l}98.00 \mathrm{a} \\
(38.03)\end{array}$ & $\begin{array}{l}91.00 \mathrm{~b} \\
(28.17)\end{array}$ & $\begin{array}{l}93.00 \mathrm{ab} \\
(30.98)\end{array}$ & $\begin{array}{l}95.00 \mathrm{ab} \\
(33.80)\end{array}$ & $\begin{array}{l}89.00 \mathrm{~b} \\
(25.35)\end{array}$ & $\begin{array}{l}98.00 \mathrm{a} \\
(38.03)\end{array}$ & $71.0 \mathrm{o} \mathrm{c}$ \\
\hline 13. & Hira-2 & $\begin{array}{l}98.00 \mathrm{a} \\
(38.03)\end{array}$ & $\begin{array}{l}91.00 \mathrm{~b} \\
(28.17)\end{array}$ & $\begin{array}{l}93.00 \mathrm{ab} \\
(30.98)\end{array}$ & $\begin{array}{l}95.00 \mathrm{ab} \\
(33.80)\end{array}$ & $\begin{array}{l}89.00 \mathrm{~b} \\
(25.35)\end{array}$ & $\begin{array}{l}98.00 \mathrm{a} \\
(38.03)\end{array}$ & $71.00 \mathrm{c}$ \\
\hline 14. & Hira-4 & $\begin{array}{l}98.00 \mathrm{a} \\
(28.95)\end{array}$ & $\begin{array}{l}91.00 \mathrm{a} \\
(19.74)\end{array}$ & $\begin{array}{l}95.00 \mathrm{a} \\
(25.00)\end{array}$ & $\begin{array}{l}92.00 \mathrm{a} \\
(21.05)\end{array}$ & $\begin{array}{l}91.00 \mathrm{a} \\
(19.74)\end{array}$ & $\begin{array}{l}96.00 \mathrm{a} \\
(26.32)\end{array}$ & $76.00 \mathrm{~b}$ \\
\hline 15. & Hira-6 & $\begin{array}{l}98.00 \mathrm{a} \\
(38.03)\end{array}$ & $\begin{array}{l}91.00 \mathrm{~b} \\
(28.17)\end{array}$ & $\begin{array}{l}93.00 \mathrm{ab} \\
(30.98)\end{array}$ & $\begin{array}{l}95.00 \mathrm{ab} \\
(33.80)\end{array}$ & $\begin{array}{l}89.00 \mathrm{~b} \\
(25.35)\end{array}$ & $\begin{array}{l}98.00 \mathrm{a} \\
(38.03)\end{array}$ & $71.00 \mathrm{c}$ \\
\hline
\end{tabular}

Data in parenthesis indicate \% increase over control.

Values within the same column having a common letter (s) do not differ significantly at $5 \%$ level of significance

$\mathrm{T}_{1}=\mathrm{BAU}-$ Biofungicide (3\%), $\mathrm{T}_{2}=$ Bavistin (0.3\%), $\mathrm{T}_{3}=$ Thiovit $(0.3 \%), \mathrm{T}_{4}=$ Provax $(0.3 \%), \mathrm{T}_{5}=$ Garlic extract $(1: 1), \mathrm{T}_{6}=$ Allamanda leaf extract (1:1) and $T_{7}=$ Control (untreated). 
The highest prevalence of seed borne fungi was found in Control and the lowest prevalence of seed borne fungi were recorded when seeds treated with BAU-Biofungicide which was closely followed by Allamanda leaf extract and Garlic extract (Table 5). Out of the treatments BAU-Biofungicide showed excellent effect in controlling seed borne fungal pathogens and extract of Allamanda, Bavistin, Provax and Thiovit were also found good for controlling seed borne pathogens of hybrid rice seeds. All the botanicals significantly reduced seed borne fungal pathogens of hybrid rice, simultaneously increased the germination percentage and growth characters of rice seedling. The present findings supported by the reports of Ahmed et al. (2013); Roy et al. (2011), Amin et al. (2009). BAU-Biofungicide is a Trichoderma based biocontrol means for controlling pathogens. Trichoderma produced a chemical, Trichodermin that inhibits the growth of fungi. Moreover, Trichoderma produces growth promoting chemical that induce plants to increase its growth.

Table 5. Effect of seed treatments on prevalence of seed borne fungi of hybrid rice seeds by Blotter method

\begin{tabular}{|c|c|c|c|c|c|c|c|c|c|c|c|}
\hline \multirow[t]{2}{*}{ Treatment } & \multicolumn{11}{|c|}{$\%$ Prevalence of seed borne fungi } \\
\hline & $\begin{array}{c}\text { Bipolaris } \\
\text { oryzae }\end{array}$ & $\begin{array}{l}\text { Fusarium } \\
\text { moniliforme }\end{array}$ & \begin{tabular}{|c|} 
Fusarium \\
oxysporum
\end{tabular} & $\begin{array}{c}\text { Aspergillus } \\
\text { flavus }\end{array}$ & $\begin{array}{c}\text { Aspergillus } \\
\text { niger }\end{array}$ & $\begin{array}{c}\text { Aspergillus } \\
\text { candidus }\end{array}$ & $\begin{array}{l}\text { Alternaria } \\
\text { padwickii }\end{array}$ & $\begin{array}{c}\text { Alternaria } \\
\text { tenuis }\end{array}$ & $\begin{array}{l}\text { Penicillium } \\
\text { spp. }\end{array}$ & $\begin{array}{l}\text { Curvularia } \\
\text { lunata }\end{array}$ & $\begin{array}{c}\text { Nigrospora } \\
\text { oryzae }\end{array}$ \\
\hline$\overline{T_{1}}$ & $1.13(100)$ & $0.00(1$ & $0.60(91.14)$ & $0.20(90.78)$ & $0.00(100.00)$ & $0.43(90.99)$ & $0.00(100.00)$ & $0.17(93.77)$ & $0.00(100.00)$ & $0.20(94.96)$ & $0.033(97.03)$ \\
\hline $\mathrm{T}_{2}$ & $2.2(76.00)$ & 0.50 & $1.3(8$ & $0.6(72.35)$ & $0.03(90.91)$ & $0.83(82.60)$ & 0.00 & & 7.00) & $2.37)$ & L.98) \\
\hline $\mathrm{T}_{3}$ & $1.86(79.72)$ & 0.30( & $1.27(81.24)$ & $0.67(69.12)$ & $0.00(100.00)$ & $0.67(\varepsilon$ & 0.00 & & .61) & 13) & .99) \\
\hline $\mathrm{T}_{4}$ & $1.96(78.63)$ & $0.00(100.00)$ & $0.97(85.67)$ & $0.63(70.97)$ & $0.00(100.00)$ & $0.67(85.95)$ & 0.00 & $0.470(82.78)$ & $0.03(98.31)$ & $4.13)$ & $0.13(88.29)$ \\
\hline$T_{5}$ & $2.56(72.08)$ & 0.23( & $1.67(75.33)$ & $1.1(49.31)$ & $0.1(69.70)$ & 1.16 & 0.00 & 0.7 & 4.35) & $7.10)$ & (84.69) \\
\hline $\mathrm{T}_{6}$ & $1.40(84.73)$ & $0.00(100)$ & $0.83(87.74)$ & $0.20(90.78)$ & $0.00(100.00)$ & $0.60(87.42)$ & $0.00(100.00)$ & $0.40(85.35)$ & $0.00(100.00)$ & $0.20(94.96)$ & $0.03(97.03)$ \\
\hline $\mathrm{T}_{7}$ & 9.17 & 1.4 & 6.77 & 2.17 & 0.33 & 4.77 & 0.70 & 2.73 & 1.77 & 3.97 & 1.11 \\
\hline
\end{tabular}

$\mathrm{T}_{1}=\mathrm{BAU}$-Biofungicide (3\%), $\mathrm{T}_{2}=$ Bavistin (0.3\%), $\mathrm{T}_{3}=$ Thiovit $(0.3 \%), \mathrm{T}_{4}=$ Provax (0.3\%), $\mathrm{T}_{5}=$ Garlic extract (1:1), $\mathrm{T}_{6}=$ Allamanda leaf extract (1:1) and $\mathrm{T}_{7}=$ Control (untreated).

Data in parenthesis indicate $\%$ decrease over control.

The vigor index of treated seeds is increased by up to $713.78 \%$ over the untreated control when seeds treated with BAU-Biofungicide (Table 6). This finding is an agreement with the findings of Hossain (2011), Bhuiyan (2005) where they found vigor index increased in BAU Bio-fungicide treated vegetable seed. Sultana et al. (2009) evaluated Bavistin treated seed showed higher vigor index (2843) followed by BAU Bio-fungicide treated seed (2661) of wheat in cv. Kanchan.

Table 6. Effect of seed treatment on increase vigor index over control of different varieties of hybrid rice by Tray method)

\begin{tabular}{|c|c|c|c|c|c|c|c|}
\hline \multirow{3}{*}{$\begin{array}{l}\text { Sl } \\
\text { No. }\end{array}$} & \multirow[t]{3}{*}{ Variety } & \multicolumn{6}{|c|}{$\%$ Vigor Index increased over control } \\
\hline & & \multicolumn{6}{|c|}{ Treatment } \\
\hline & & $\mathrm{T}_{1}$ & $T_{2}$ & $T_{3}$ & $\mathrm{~T}_{4}$ & $T_{5}$ & $T_{6}$ \\
\hline 1. & Durber & +214.08 & +89.99 & +188.79 & +105.73 & +137.99 & +163.97 \\
\hline 2. & Agomoni & +216.42 & +84.44 & +182.34 & +106.06 & +127.84 & +153.74 \\
\hline 3. & Meghna & +282.40 & +151.02 & +231.27 & +164.99 & +197.35 & +217.15 \\
\hline 4. & Hybrid super & +257.93 & +137.88 & +217.23 & +160.05 & +177.57 & +196.81 \\
\hline 5. & Aloron & +202.50 & +77.79 & +143.89 & +82.40 & +109.20 & +164.23 \\
\hline 6. & Jagoron & +389.78 & +228.03 & +346.21 & +247.95 & +277.81 & +296.92 \\
\hline 7. & Moyna & +468.84 & +288.75 & +550.82 & +332.78 & +328.36 & +342.45 \\
\hline 8. & Tia & +633.78 & +378.62 & +616.79 & +462.79 & +478.78 & +490.58 \\
\hline 9. & Gold & +713.78 & +427.99 & +681.97 & +507.09 & +533.51 & +546.84 \\
\hline 10. & Safollo & +363.93 & +236.98 & +362.06 & +290.01 & +293.89 & +270.55 \\
\hline 11. & Suborno & +435.70 & +270.13 & +422.53 & +328.20 & +327.77 & +305.82 \\
\hline 12. & Hira-1 & +311.60 & +181.43 & +314.70 & +208.12 & +219.09 & +244.87 \\
\hline 13. & Hira-2 & +299.58 & +179.25 & +323.68 & +200.37 & +212.60 & +236.90 \\
\hline 14. & Hira-4 & +326.73 & +107.88 & +290.63 & +153.87 & +203.80 & +244.2 \\
\hline 15. & Hira-6 & +329.34 & +198.74 & +336.90 & +222.66 & +236.56 & +259.84 \\
\hline
\end{tabular}

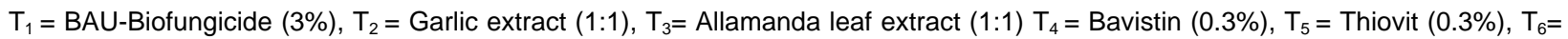
Provax (0.3\%), and $\mathrm{T}_{7}=$ Control (untreated). 


\section{Conclusion}

Based on the findings of the present study it may be concluded that among different seed treatments BAU-Biofungicide (3\%) has been found as superior for controlling different seed borne fungi, increasing germination, vigor index, improving quality of hybrid seeds of rice and might be considered as effective, economic and eco-friendly disease management strategy of hybrid rice grown in Bangladesh.

\section{References}

Ahmed, M., Hossain, M., Hassan, K., and Dash, C.K. 2013. Efficacy of different plant extract on reducing seed borne infection and increasing germination of collected rice seed sample. Unil. J. Plant Sci., 1(3): 66-73.

Akter, M.A., Hasan, A.K.M.K, Uddin, S.A. and Hossain, I. 2015. Seed treatment for improving quality of hybrid seeds of rice. Asian J. Medic. Bio. Res., 1 (3): 406-415.

Amin, R.B.M., Rashid, M.M. and Meah, M.B. 2009. Efficacy of garlic tablet to control seed-borne fungal pathogens of cucumber. J. Agri. Rural Dev., 7(1 and 2): 135-138.

Baki, A.A. and Anderson, J.D. 1972. Physiological and biological deterioration of seeds. Seed Biology II Academic press, New York pp. 283-315.

Bhuiyan, A.B. 2005. BAU-Biofungicides Controlling Seedling Diseases of Winter Vegetables, MS Thesis, Department of Plant Pathology, Bangladesh Agricultural University, Mymensingh.

Chowdhury, M.M.H. 2012. Evaluation of quality status and management Truthfully Labeled Seed (TLS) of rice in Bangladesh, Ph.D Thesis, Department of Seed Science and Technology, Bangladesh Agricultural University, Mymensingh.

Chowdhury, M.M.H., Hossain, I., Day, P., Ahmed, M. and Mahmud, H. 2013. Effect of seed sources and seed treatments on disease incidence, severity and seed yield of rice in Bangladesh. J. Agrofores. Envn. 7 (2): 23-27.

Fakir G.A. 2000: An annotated list of seed-borne disease in Bangladesh. Seed Pathology Laboratory. Department of Plant Pathology, BAU, Myrnensingh. pp. 41.

Fakir, G.A., Hossain, I., Ahmed, M.U., Anam, M.K., Islam, M.N. and Rahman, M. 2003. Effect of ash, chalk powder and Neem leaf on quality of Boro rice seed stored in gunny bag, motka, plastic drum and tin jar. Review and planning Meeting of the Seed Health Improvement, BRRI, Gazipur, Bangladesh, 21-22 April 2002.

Fakir, G.A., Hossain, I., Ahmad, M.U., Anam, M.K., Islam, M.N. and Rahman, M. 2002. Quality of farmers Boro and T. Aman rice seeds collected before sowing from Bogra, Rajshahi and Rangpur districts of Bangladesh. Review and Planning meeting of the Rice Seed Health Improvement BRRI, Gazipur, Bangladesh 17-18 April.

Haque, A.H.M.M., Akon, M.A.H., Islam, M.A., Khalequzzaman, K.M. and Ali, M.A. 2007. Study of seed health, germination and seedling vigor of farmers produced rice seeds. Int. J. Sustain. Crop Prod., 2(4): 34-39.

Hossain, M.H. and Hossain, I. 2012. Effect of seed treatment with different botanicals, Bavistin and BAU Bio-fungicide on germination and seedling vigor of groundnut. Bangladesh Agron. J., 16 (1): 87-94.

Hossain, I. 2011. Unique ecofriendly means and new dimension of plant disease control in Bangladesh. Department of Plant Pathology, Bangladesh Agricultural University, Mymensingh., Bangladesh.

Hossain I, Dey, P. 2011: Annual Report. Seed Pathology Centre, BAU, Mymensingh, Bangladesh. pp. 5-6

Howlader, M.T.H. 2003. Prevalence of insect pests in rice seed and its loss assessment as influenced by storage structures at Mymensingh Sadar Upazilla Mymensingh. Department of Entomology, Bangladesh Agricultural University, Mymensingh.

ISTA, 2001. International Rules for seed Testing Association, 31: 107-115.

ISTA, 1996. International Rules for Seed Testing. International Seed-Testing Association, Seed Science and Technology, $24: 39-42$.

Nahar, K., Islam, M.M. and Hossain, I. 2004. Study on seed health status of farmers saved rice seed cv. BR 1 from Bogra. Bangladesh J. Seed Sci. Tech., 8 (1\&2): 1-5.

Naznin, H. and Hossain, I. 2004. Effect of BAU-Biofungicide on germination and seedling vigor of some summer vegetables. Bangladesh J. Seed Sci. Tech., 8 (1-2): 85-90.

Ora, N.A.N., Faruq, M.T., Islam, N., Akhtar and Rahman, M.M. 2011. Detection and identification of seed borne pathogens from some cultivated hybrid rice varieties in Bangladesh. Middle-East J. of Sci. Res. , 10 (4) : 482-488. 
Roy, K.K., Khan, M.M.R., Hossain, M.M. and Khokon, M.A.R. 2011. Feasibility of quality improvement of jute seed by plant extracts. Progressive Agric., 22(1 \& 2): 1 - 10.

Saranraj, P., Sivasakthivelan, P., Sivasakthi, S. 2013. Prevalence and production of plant growth promoting substance by pseudomonasfluorescens isolated from paddy rhizophere soil of Cuddalore district, Tamil Nadu, India. African J. of Basic and App. Sci., 5: 95-101.

Sultana, R., Hossain, I., Ahmed, S. and Mamun, M.A.A. 2009. Efficacy of BAU-Biofungicide in controlling leaf spot of Wheat (Triticum aestivitm). Eco-friendly Agri. J., 22: 392-395.

Uddin, M.J. 2005. The quality of farmers stored rice seed of Begumgonj Upazilla, M. S. Thesis, Department of Plant Pathology, Bangladesh Agricultural University, Mymensingh. 